\title{
Stationary and axisymmetric magnetized equilibria of stars and winds
}

\author{
Shin Yoshida ${ }^{1}$, Kotaro Fujisawa ${ }^{1}$, Yoshiharu Eriguchi ${ }^{1}$, \\ Shijun Yoshida ${ }^{2}$ and Rohta Takahashi ${ }^{3}$ \\ ${ }^{1}$ Department of Earth Science and Astronomy, Graduate School of Arts and Sciences, \\ University of Tokyo \\ Komaba, Meguro-ku, Tokyo 153-8902, Japan \\ email: yoshida@ea.c.u-tokyo.ac.jp \\ ${ }^{2}$ Astronomical Institute, Tohoku University \\ 6-3 Aramaki, Aoba-ku, Sendai 980-8578, Japan \\ ${ }^{3}$ Cosmic Radiation Laboratory, The Institute of Physical and Chemical Research, RIKEN, 2-1 \\ Hirosawa, Wako, Saitama 351-0198, Japan
}

\begin{abstract}
We present a new formulation to compute numerically stationary and axisymmetric equilibria of magnetized and self-gravitating astrophysical fluids. Under the assumption of ideal MHD, the stream function for the flow can be chosen as a basic variable with which the EulerMaxwell equations are cast into a set of basic equations, i.e. a generalized Bernoulli equation and a Grad-Shafranov-like equation by employing various integral conditions. A novel feature of this formulation is that systems with stars, disks and winds are treated in a simple unified picture and the magnetic field structures can contain both poloidal and toroidal components.
\end{abstract}

Keywords. stars: magnetic fields, stars: rotation, stars: winds, outflows

\section{Introduction}

From the scale of the solar surface activities to those of galactic nuclei, magnetic field is a very important factor in astrophysical processes. Many activities of stars, such as flares, high energy photon emission or stellar winds, are thought to be driven by their magnetic field. Evolution of a molecular cloud as a birth place of a star is affected by the presence of magnetic field, while the proto-stellar activity is mediated by magnetic field. Accretion processes onto compact objects and outflows from them are thought to be driven/affected by magnetic field.

Although in reality outflows/inflows from magnetized objects are time-dependent and asymmetric, many of the objects are regarded to possess well-defined time-averaged states with axisymmetry. Therefore a theoretical modeling of stationary and axisymmetrtic structure in magnetized objects has been an important issue in astrophysics. The stationary solutions of magnetized objects, however, has been rather difficult to obtain, especially with the out/inflow. Numerical solutions so far obtained are that of Weber \& Davis (1967), Pneuman \& Kopp (1971) and Sakurai (1985, 1987), the last two of which are the only ones of self-consistent treatment. Most of the other solutions of (quasi-) stationary states are obtained by long-time numerical MHD simulations (cf. Keppens \& Goedbloed, 1999). This method has an advantage of avoiding apparent singularity of equations related to critical points of flows, while it is rather difficult to study a structure of flow and magnetic field in a large scale by MHD simulations. Thus an important problem of collimation and acceleration of astrophysical winds and jets may not be fully solved by this method (at least by the present computational resources). We therefore 
develop a new numerical scheme that solves stationary and axisymmetric out/inflows around magnetized stars/disks. The new scheme solves integrated form of the equation of motion of gas, rather than a differential form of it. This makes us easier to spot and handle critical points of flow which are not known a priori, but obtained only after the system of equations are solved.

\section{Formulation}

We assume the system to be stationary and axisymmetric. Fluid in the system is perfect, isentropic. The conductivity of it is infinite and the electromagnetic field follows that of ideal MHD

$$
E^{a}+\frac{1}{c} \epsilon^{a b c} v_{b} B_{c}=0
$$

where $\epsilon^{a b c}$ is Levi-Civita tensor and the other symbols have their usual meanings. Basic equations are 1) Continuity equation, 2) four of Maxwell's equations, 3) equation of hydromagnetic momentum balance and 4) Poisson's equation for gravity. From the assumption of stationarity and axisymmetry with 1) Continuity equation, we can define a stream function $Q$ in such a way that the meridional velocity components are expressed as

$$
v^{R}=-\frac{1}{\rho R} \frac{\partial Q}{\partial z}, \quad v^{z}=\frac{1}{\rho R} \frac{\partial Q}{\partial R}
$$

in cylindrical polar coordinate $(R, \varphi, z)$. The solenoidal condition of magnetic field from Maxwell's equation implies similar expression of magnetic field as a scalar magnetic flux function $\Psi$, which is used in an alternative formalism of magnetized flow (Lovelace et al. (1987); see also Fujisawa et al. in this volume). From the ideal MHD condition it can be shown that the magnetic flux function is a functional of $Q$. On the other hand, other components of the same condition imply that there is a relation

$$
\frac{B_{\varphi}}{\rho R^{2}}-\frac{d \Psi}{d Q} \frac{v_{\varphi}}{R^{2}}=\sigma[Q]
$$

where $\sigma$ is an arbitrary functional of $Q$. From the toroidal component of the momentum equation we have

$$
v_{\varphi}-\frac{1}{4 \pi} \frac{d \Psi}{d Q} B_{\varphi}=\ell[Q],
$$

where $\ell$ is an arbitrary functional of $Q$. Another arbitrary function $\nu$ of $Q$ is shown to exist by examining a curl of momentum equation

$$
v^{\varphi} \frac{B^{\varphi}}{4 \pi} \frac{d^{2} \Psi}{d Q^{2}}+v^{\varphi} \frac{d \ell}{d Q}+\frac{1}{4 \pi} \frac{d \sigma}{d Q} B_{\varphi}-\frac{\zeta^{\varphi}}{\rho}+\frac{j^{\varphi}}{c \rho} \frac{d \Psi}{d Q}=\nu[Q],
$$

where $\vec{\zeta}$ is vorticity of the flow and $\vec{j}$ is current density.

From the meridional components of the momentum equation has a first integral

$$
\int^{Q} \nu(q) d q-\frac{B_{\varphi}}{4 \pi} \sigma-\int^{p} \frac{d p^{\prime}}{\rho}-\Phi-\frac{1}{2 \rho^{2} R^{2}}|\nabla Q|^{2}-\frac{1}{2} v_{\varphi} v^{\varphi}=C \text { (const.), }
$$

where $\Phi$ is the gravitational potential satisfying Poisson's equation

$$
\triangle \Phi=4 \pi G \rho .
$$



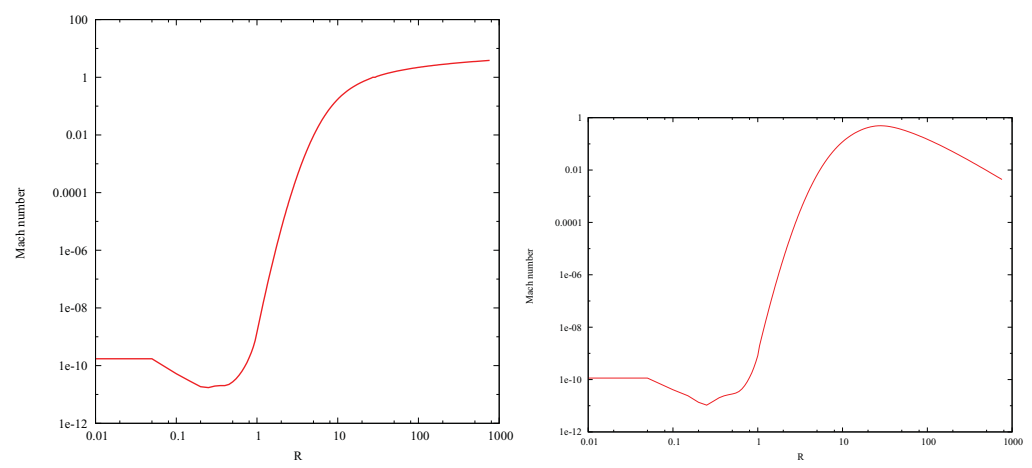

Figure 1. Profiles of Fast-wave Mach number for wind (left panel) and breeze (right panel) solutions.

From the definition of vorticity in $\varphi$, we have a Poisson-like differential equation for $Q$

$$
\triangle\left(\frac{Q}{R} e^{i \varphi}\right)=e^{i \varphi}\left(\frac{\nabla \rho}{\rho} \cdot \frac{\nabla Q}{R}-R \zeta^{\varphi}\right)
$$

These two Poisson PDEs are formally integrated by using proper Green's function as

$$
\Phi=-G \int \frac{\rho\left(\overrightarrow{r^{\prime}}\right)}{\left|\vec{r}-\overrightarrow{r^{\prime}}\right|} d V^{\prime}
$$

and

$$
Q=-e^{-i \varphi} \frac{R}{4 \pi} \int \frac{S_{Q}\left(\overrightarrow{r^{\prime}}\right)}{\left|\vec{r}-\overrightarrow{r^{\prime}}\right|} d V^{\prime}
$$

where $S_{Q}$ is the right hand side of Eq.(2.8).

By specifying functionals of $Q(\Psi, \sigma, \ell, \nu)$, we iteratively solve Eq. (2.6), Eq. (2.9) and Eq.(2.10) .

\section{Results}

In the following we show some of the preliminary results obtained by our numerical code to compute fluid flow and magnetic field structure of rotating stars. The equation of state of the gas is assumed to be polytropic ones, whose indices are $N=3$ inside the star and $N=20$ outside, respectively. The stellar surface is defined by $\rho / \rho_{c}=10^{-3}$ where $\rho_{c}$ is the central density.

We show two kinds of solution here. in Fig.1, the Mach number (defined to be the ratio of poloidal velocity component to poloidal fast-wave velocity) is plotted as a function of radial distance from the center of the star. On the left panel, the Mach number increases from the surface of the star $(r=1)$ and exceeds unity at $r \sim 30$. This solution corresponds to the proper "wind" solution. On the right panel we have a solution whose Mach number always stays below unity. This solution is so-called "breeze" solution.

In Fig. 2 two typical profiles of stream lines (magnetic field lines) in the meridional section of the star are plotted. With different choices of the functionals of $Q$, we have complex field structure inside the star, though the flow pattern far out may be close to Parker's solution of the solar wind (Parker, 1958). In this example, the magnetic field is rather weak and the structure outside the star looks very close to the Parker's solution. 

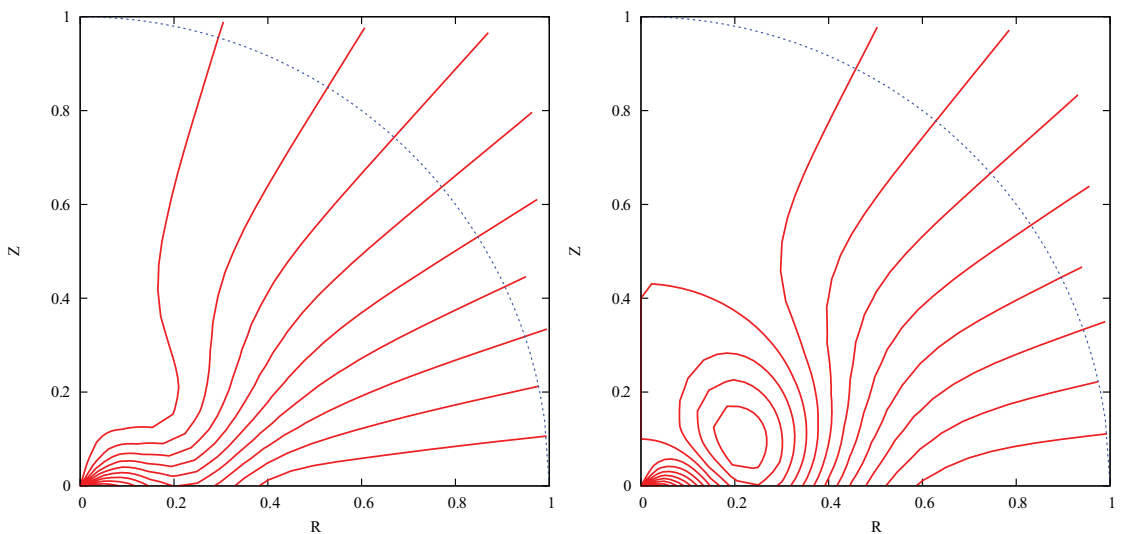

Figure 2. Examples of wind (left) and breeze (right) solutions. Stream lines (magnetic field lines) in the meridional section of a star shown as a solid lines. Blue dashed line corresponds to the surface of the star (defined as a isobaric surface of $\rho / \rho_{c}=10^{-3}$ ).
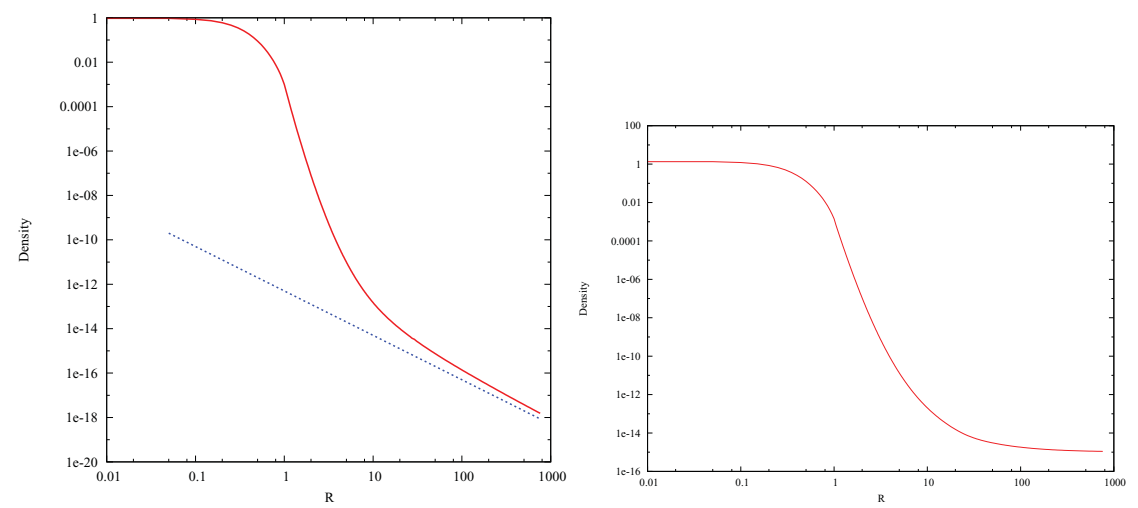

Figure 3. Density profiles of the wind (left) and the breeze (right) solutions. As the wind solution here tends asymptotically to Parker's solution far out the star, the density distributions falls as $\sim r^{-2}$, while that of the breeze solution does not.

In Fig. 3 we show density profile of wind/breeze solutions. The wind solution (left panel) tends to a Parker's solution far outside, with a density $\rho \sim r^{-2}$, while the breeze solution (left panel) tends to a constant density as expected.

\section{References}

Fujisawa, K., Yoshida, S., \& Eriguchi, Y. 2010, MNRAS, submitted

Keppens, R. \& Goedbloed, J. P. 1999, A \& A, 343, 251

Lovelace, R. V. E., Mehanian, C., Mobarry, C. M., \& Sulkanen M. E. 1986, ApJS, 62, 1

Parker, E. N. 1958, ApJ, 128, 664

Pneuman, G. W. \& Kopp, R. A. 1971, Solar Phys., 18, 258

Sakurai, T. $1985, A \& \mathcal{E} A, 152,121$

Sakurai, T. 1987, PASJ, 39, 821

Weber, E. J. \& Davis, L. 1967, ApJ, 148, 217 